Cahiers d'études africaines

\title{
Dahou, Tarik. - Entre parenté et politique
}

\section{Charlotte Pezeril}

\section{(2) OpenEdition}

Journals

Édition électronique

URL : http://journals.openedition.org/etudesafricaines/14280

DOI : 10.4000/etudesafricaines. 14280

ISSN : 1777-5353

Éditeur

Éditions de l'EHESS

\section{Édition imprimée}

Date de publication : 8 septembre 2011

Pagination : 704-707

ISBN : 978-2-7132-2298-6

ISSN : 0008-0055

\section{Référence électronique}

Charlotte Pezeril, «Dahou, Tarik. - Entre parenté et politique », Cahiers d'études africaines [En ligne], 202-203 | 2011, mis en ligne le 10 octobre 2011, consulté le 21 septembre 2020. URL : http:// journals.openedition.org/etudesafricaines/14280 ; DOI : https://doi.org/10.4000/etudesafricaines. 14280

Ce document a été généré automatiquement le 21 septembre 2020.

(C) Cahiers d'Études africaines 


\title{
Dahou, Tarik. - Entre parenté et politique
}

\author{
Charlotte Pezeril
}

\section{RÉFÉRENCE}

DAHOU, Tarik. - Entre parenté et politique. Développement et clientélisme dans le Delta du Sénégal. Préface de Jean Copans. Paris, Karthala; Dakar, Enda Graf Sahel, 2004, 364 p., bibl., gloss.

1 Alors que les politiques de libéralisation mises en place au Sénégal dans les années 1980 laissaient présager la substitution des rapports marchands aux rapports clientélistes, Tarik Dahou démontre qu'il n'en est rien. Le clientélisme s'est renouvelé sur de nouvelles bases productives. L'originalité de l'analyse est de partir des travaux de l'anthropologie politique pour déconstruire les notions de clientélisme et de factionnalisme, tout en s'insérant dans la réalité sénégalaise. Selon lui, «le factionnalisme sénégalais doit être considéré comme le lieu de rencontre de logiques politiques étatiques et de logiques politiques lignagères» (p.22). L'importance de ces dernières est telle que l'individu peut en arriver à sacrifier son intérêt personnel pour préserver sa famille factionnelle. Sur ce point (notamment), Tarik Dahou prend ses distances avec le courant anthropologique des «courtiers du développement» (Olivier de Sardan, Bierschenk \& Chauveau 2000) ${ }^{1}$ et plus particulièrement avec Giorgio Blundo $(1995,1998)^{2}$ sur le Sénégal en relativisant le principe utilitariste des stratégies factionnelles. L'insertion dans une faction n'est pas seulement un moyen pour obtenir des biens et des faveurs, il permet aussi de tenir son rôle, de se conformer aux normes parentales et d'y affirmer son identité sociale.

2 L'intérêt de son ouvrage est loin de s'arrêter là ${ }^{3}$. À partir d'une minutieuse enquête de terrain effectuée dans un village de la vallée du fleuve Sénégal, Tarik Dahou analyse les diverses relations de dépendance entre villageois et leurs multiples liens de dette. Dans une première partie, il revient sur l'histoire des rapports entre l'État et la paysannerie 
en général avant d'aborder plus précisément la situation dans le Delta. Il montre que les politiques économiques successives ont permis de maintenir l'hégémonie du pouvoir central, sans assurer la progression des revenus paysans. La première phase de libéralisation (initiée par les Plans d'ajustement structurels dès 1980), même si elle a favorisé l'émergence des organisations paysannes et la «responsabilisation» des agriculteurs, a aggravé la situation en faisant peser sur eux le poids des aménagements (barrages, réseaux hydrauliques) nécessaires pour contrer la sécheresse et installer l'irrigation comme mode principal de culture. Avec la seconde phase de libéralisation début 1990, les Groupements d'intérêt économique, structures juridiquement souples permettant de demander officiellement des crédits, se sont multipliés, aboutissant à l'endettement des exploitants et à la banqueroute des institutions financières en 1995, un an après la dévaluation du FCFA qui a fait s'écrouler la filière rizicole. Aux anciens risques climatiques s'ajoutent les risques marchands. Finalement, les financements des bailleurs ont conduit à l'établissement de nouvelles clientèles dans le milieu rural. En effet, la fragilité des institutions paysannes les rend perméables aux clivages partisans. La course aux terres, distribuées par l'État, et les concurrences entre fédérations paysannes exacerbent les tensions. Ainsi, «les besoins de sécurisation des exploitants sont palliés par la mise en branle de réseaux clientélistes» (p.102). Mais comment fonctionnent ces réseaux?

3 L'auteur y répond dans la seconde partie de l'ouvrage en abordant le clientélisme à partir des relations (et non des institutions) politiques et lignagères. Dans ce cadre, le concept de factionnalisme lui semble davantage pertinent dans la mesure où il articule ces deux niveaux et peut se définir comme un «système d'opposition dual où les ressources circulent au sein d'une clique» au détriment d'une autre (p.122). Le village est un espace de solidarités et de concurrences: d'un côté, de multiples dons y circulent, particulièrement au sein du lignage utérin; de l'autre, les factions politiques peuvent attirer des groupes d'affins (sans règle de filiation clairement identifiées). Les luttes n'empêchent toutefois pas le village d'être une entité politique disposant d'assemblées collectives (mboloo) chargées de régler les conflits. Le contexte villageois ne se caractérise pas par une absence d'institutionnalisation du politique. «Plutôt que de raisonner en terme d'espace public, sans doute, vaudrait-il mieux parler d'espace commun, au sein duquel les logiques lignagères [et factionnelles, pourrait-on rajouter] sont transcendées au profit de l'intérêt général du village» (p.146).

Quelles sont les relations de dépendance prégnantes aujourd'hui dans le village? Tout d'abord, celles entre aînés et cadets qui, bien qu'ayant évolué, sont toujours vivaces. La crise économique et les importantes migrations modifient les structures familiales, en poussant les cadets à trouver eux-mêmes des sources de financement (souvent extraagricoles) et à négocier directement avec les institutions de développement (s'ils ont suivi une scolarité). Mais l'augmentation des risques productifs limite la quête d'autonomie des cadets, qui restent liés par des relations de dette même en dehors de la concession. Par ailleurs, l'émergence de nouveaux leaders, maîtrisant le rapport aux institutions de crédit, multiplie les liens de clientèle. Ces leaders peuvent faire appel à de la main-d'œuvre, leur assurant une part de la production et s'assurant en échange de leur appui politique. «Il n'existe aucune réciprocité marchande dans un tel lien [...], car il ne s'agit pas d'un échange entre équivalents» (p.192). À travers divers exemples, Dahou montre néanmoins que les stratégies des exploitants face aux leaders sont diverses et dépendent de leur "capital parental», politique ou économique dans le village. L'auteur ne prend toutefois pas suffisamment en compte, à mon sens, les 
allégeances religieuses, même s'il précise le cas d'un exploitant parvenant à obtenir des financements auprès de son marabout mouride (pp.200-201), ce qui lui permet, en tant que cadet, de s'émanciper pendant un temps de sa famille et de ses obligations de redistribution. Bien que la situation du Delta diffère certainement sur ce point de celle de la région arachidière, les liens marabout-disciple sont aussi traversés par des liens de dette (Pezeril 2008) 4 et les alliances lignagères maraboutiques participent aux luttes factionnelles politiques. Par exemple, dans le village de Touba Fall, la «capitale Baay Faal» située à une dizaine de kilomètres de Touba, les divergences entre lignages Palène (descendants de Cheikh Ibra Fall) ont abouti à la nomination de deux chefs de village représentants chacun un lignage maraboutique. En outre, les liens de dette existent entre condisciples, entre mbokk-taalibe et permettent de mieux comprendre certaines alliances ou conflits inter ou intra familiaux. Par dessus tout, les liens de dette me semblent devoir être analysés en tant que logique sociale, et pas seulement à travers l'effectivité des échanges de biens, de services et de personnes. Dans cette logique, donner, échanger, prêter sont nécessaires pour recevoir. Chacun est imbriqué dans un faisceau de dettes personnelles, familiales et lignagères. Cependant, si cette logique permet une certaine redistribution, elle reste inégalitaire et toujours régie par les rapports de dépendance entre aînés et cadets, entre hommes et femmes, entre patrons et clients, entre marabouts et disciples. Mais de même que le rapport de soumission du disciple au marabout doit être relativisé - ne serait-ce que dans la mesure où le disciple peut changer de marabout, en avoir plusieurs ou encore gérer de façon flexible sa relation et décider de sa teneur et de sa régularité (Pezeril 1997) ${ }^{5}$ - «les dépendants n'entretiennent pas forcément des rapports stables avec leur leader» et peuvent en changer en restant dans la parenté (p.206). Il n'empêche que la libéralisation a entraîné le passage "d'un clientélisme bureaucratique à un clientélisme capable de mobiliser les ressources de la sphère privée pour les utiliser dans les luttes factionnelles» (ibid.). Les alliances se sont diversifiées, mais les liens parentaux restent déterminants et se combinent aux liens clientélistes pour contrôler les paysans.

Ensuite, à travers l'étude des organisations villageoises (foyer des jeunes et sections villageoises) et de leur mode de désignation des leaders, Dahou rend compte du contrôle social pesant sur les dépendants dans la mesure où ils ne peuvent exprimer publiquement leur défiance vis-à-vis du leader. D’ailleurs le vote secret est jugé «contre nature» puisque son issue divise le groupe. «L'absence de visibilité du pacte d'alliance affaiblit la réciprocité du lien» (p.225) et finalement diminue la légitimité de l'élu, alors que l'élection à main levée permet négociations et affermissement de la confiance. Les conflits ne pouvant s'exprimer publiquement, ils passent souvent par la rumeur qui s'avère un moyen de réaffirmation ou de redéfinition des normes de comportement. Les cadets fustigent la régulation parentale et militent pour des règlements basés sur l'équité des droits (p.258). Mais les organisations villageoises sont instables et la renégociation des règles internes permanente. Elles sont en tous les cas devenues les «principales arènes de la compétition entre factions» (p.261), banalisant les pratiques de corruption. Ces dernières ne permettent pas seulement d'accaparer des ressources, mais d'acquérir un prestige social bénéficiant à l'individu, à sa famille et à sa faction. L'auteur s'inscrit dans une "anthropologie de l'honneur» pour comprendre ce phénomène: «L'accaparement est alors considéré comme une manière de ravir ce qui est convoité par la faction adverse. [...] Celui qui n'oserait affronter par de telles armes la faction opposée passerait au contraire pour un lâche» (p.271). Le leader est censé accumuler les ressources afin de les redistribuer, sous peine d'ostracisme social voire 
de représailles magiques. Malgré l'émergence de certaines critiques, les pratiques corruptives s'amplifient donc face à la faiblesse économique et institutionnelle de l'État.

6 Dans la dernière partie, Dahou analyse plus précisément les conflits traversant la société villageoise. Il montre que l'alliance opérée dans la première phase de libéralisation entre notables villageois et jeunes leaders se fragilise lorsque les ressources s'amenuisent et que la filière se marchandise. «Les individus peuvent désormais construire leurs cercles d'allégeance principalement à partir d'une accumulation économique, ce qui disqualifie plus rapidement les élites locales» (p.289). De plus, les normes d'honnêteté et de probité sont «dorénavant», selon l'auteur, susceptibles de surpasser les alliances lignagères dans le choix des leaders. Par exemple, le président de l'Union locale a été désigné alors qu'il n'est pas originaire du village et n'a pas de positionnement factionnel. Son impartialité et sa capacité de négociation avec les institutions de développement ont été opposées aux précédents "gaspillages» des richesses. «La nouvelle légitimité impose aux élites le choix de leaders qui ne sont pas stigmatisés pour leur comportement somptuaire» (p.298). Mais est-ce bien nouveau? Les Mourides ont toujours condamné la richesse ostentatoire parce qu'elle «développe la paresse, fait oublier ses devoirs et tente l'envie» (Wade 1987: 27) ${ }^{6}$. L'argument de moralité n'est pas récent. Ce qui semble reproché aujourd'hui aux leaders, c'est davantage leur individualisme ou égoïsme, c'est-à-dire leur nonsoumission aux normes de redistribution, que leur accaparement des ressources. Les deux «contestataires» du village remettent en cause les hiérarchies villageoises et les mécanismes de conciliation et d'euphémisation des conflits (passer par un intermédiaire, ne pas nommer «l'accusé», ne pas le défier publiquement). Ces individus ne sont toutefois pas mis à l'écart du village et leurs comportements de défiance au sein des organisations politiques cèdent la place dans la vie quotidienne à une déférence plus conforme aux comportements villageois.

7 Le pouvoir des élites locales (à l'exception des élites religieuses précise-t-il) s'est donc érodé, laissant davantage de place à la délibération et aux choix individuels. Cependant, «la rencontre des stratégies individuelles des entrepreneurs politiques et des stratégies collectives de sécurisation de la production par les lignages a produit une continuité entre un clientélisme "familial" et un clientélisme "marchand"» (p.329). La dichotomie public-privé, qui recoupe les logiques étatiques et communautaires, s'estompe finalement pour souligner les modalités opératoires du processus démocratique villageois. Cet ouvrage apporte ainsi un éclairage riche et inédit des liens sociaux actuels au Sénégal, de l'impact des différentes politiques de libéralisation et des théories de l'anthropologie politique et de ses analyses du factionnalisme et du développement en Afrique. 


\section{NOTES}

1. J.-P. OlivieR De SARDAN, T. BIERSCHENK \& J.-P. ChAUVEAU, Les courtiers du développement, Paris, Karthala, 2000.

2. G. BLUNDO, «Les courtiers du développement en milieu rural sénégalais», Cahiers d'Études africaines, 137, XXXV (1), 1995, pp.73-99; Élus locaux, associations paysannes et courtiers du développement au Sénégal, Thèse de doctorat, Lausanne, Université de Lausanne, 1998.

3. Voir également à ce propos l'intéressante mise en perspective de Jean Copans dans sa préface.

4. C. PEZERIL, Islam, marginalité et mysticisme: les Baay Faal du Sénégal, Paris, L'Harmattan («Anthropologie critique»), 2008.

5. C. PEZERIL, La relation marabout-disciple dans la confrérie mouride du Sénégal, DEA Paris, EHESS, 1997.

6. A. WADE, La doctrine économique du mouridisme, Dakar, publication privée, 1970. 